\title{
THE INVESTIGATION OF AN OUTBREAK OF CRYPTOSPORIDIOSIS IN NEW SOUTH WALES IN 2005
}

\section{Megan Black}

NSW Public Health Officer Training Program

NSW Department of Health

\section{Jeremy McAnulty}

Communicable Diseases Branch

NSW Department of Health

In April 2005 the Communicable Diseases Branch at the NSW Department of Health noticed an increase in the number of cases of cryptosporidiosis reported across NSW. This article describes how this outbreak of cryptosporidiosis was investigated and analysed.

Cryptosporidiosis is caused by the protozoan parasite Cryptosporidium and the symptoms include watery diarrhoea, abdominal cramping, loss of appetite, fever, vomiting and nausea. ${ }^{1}$ The incubation period for cryptosporidiosis is one to 12 days. Treatment is supportive only; however, most healthy people tend to recover within two to 26 days. ${ }^{2}$ An infected person will excrete infectious oocysts for several weeks after their symptoms resolve. ${ }^{2}$

Cryptosporidiosis is spread by the faecal-oral route. Transmission can be person-to-person, animal-to-person, waterborne and, in rare cases, foodborne ${ }^{1}$. Cryptosporidium oocysts are small $(4-6 \mu \mathrm{m})$ and can survive under adverse environmental conditions, including chlorination. ${ }^{1,2}$ Outbreaks of cryptosporidiosis are usually associated with contaminated drinking water supplies and swimming pools. ${ }^{1}$ To control Cryptosporidium in public swimming pools it is recommended that pools are 'superchlorinated' or 'shock-dosed' at least fortnightly. ${ }^{3}$

In 1996 cryptosporidiosis became notifiable by laboratories in NSW under the NSW Public Health Act 1991. Laboratories are required to notify public health units of positive cases of cryptosporidiosis; the public health units, in turn then enter information about the cases into the NSW Notifiable Diseases Database within three days of receiving this notification. The Communicable Diseases Branch uses the Notifiable Diseases Database to monitor the number of cases of cryptosporidiosis in NSW.

We investigated this outbreak using epidemiological, environmental and laboratory methods to determine the likely cause of the outbreak and develop public health response strategies.

\section{METHODS}

A case was defined as a person with laboratory-confirmed cryptosporidiosis notified between 1 May 2005 and 12 August 2005, with an onset date of illness after 31 March 2005. The Notifiable Diseases Database for NSW contained basic demographic information for all the cases of cryptosporidiosis notified during this period.
To investigate these cases further we designed a data collection form that included questions seeking additional demographic information about possible exposures during the 12 days before the onset of symptoms. Public health units were asked to contact the cases and interview them using this form, and to return the completed forms to the Communicable Diseases Branch. The data was entered into a central database, and the demographic characteristics and risk factors for disease were analysed.

In June 2005 an environmental investigation was undertaken that involved testing swimming pool water for Cryptosporidium oocysts. Pools selected for testing were those where more than two cases had reporting swimming during their exposure period and where at least one of these had reported onset of illness within 10 days of swimming in the pool in the month of June 2005. Environmental health officers collected approximately 100 litres of water from each pool. The water was collected in 10 litre bladders from different locations around the perimeter of the pool and this sample was then sent to Sydney Water to identify and enumerate the number of Cryptosporidium oocysts. Environmental health officers also asked about superchlorination practices at the associated pools and reviewed pool disinfectant records for documentation of superchlorination practices.

We also reviewed laboratory stool testing procedures because of anecdotal reports that laboratories were increasing the number of stools being tested for Cryptosporidium. The laboratory investigation involved contacting a convenient sample of laboratories about changes in their stool testing procedures for Cryptosporidium. A private metropolitan laboratory, a public metropolitan laboratory and a public regional laboratory were contacted. Medical Benefits Scheme changes relevant to the laboratory diagnosis of cryptosporidiosis were also reviewed, and we also analysed Medicare data for item number 69336 (which pertains to stool tests for ova, cysts and parasites), by accessing the Medical Benefits Scheme health statistics on the Medicare Australia website. ${ }^{4}$

\section{RESULTS}

\section{Epidemiological investigation}

There were 254 cases of cryptosporidiosis notified from 1 May to 12 August 2005 with an onset date after 31 March 2005. Figure 1 displays the number of cases of cryptosporidiosis reported in NSW from January 2001 to September 2005. A rise in notifications can be seen each summer. In March 2005 the number of cases continued to increase beyond the summer months, and peaked in April 2005. Notification rates were more than three times higher than expected for that time of the year. 


\section{FIGURE 1}

NUMBER OF CASES OF LABORATORY-CONFIRMED CRYPTOSPORIDIOSIS IN NEW SOUTH WALES FOR EACH MONTH FOR THE PERIOD JANUARY 2001 TO SEPTEMBER 2005

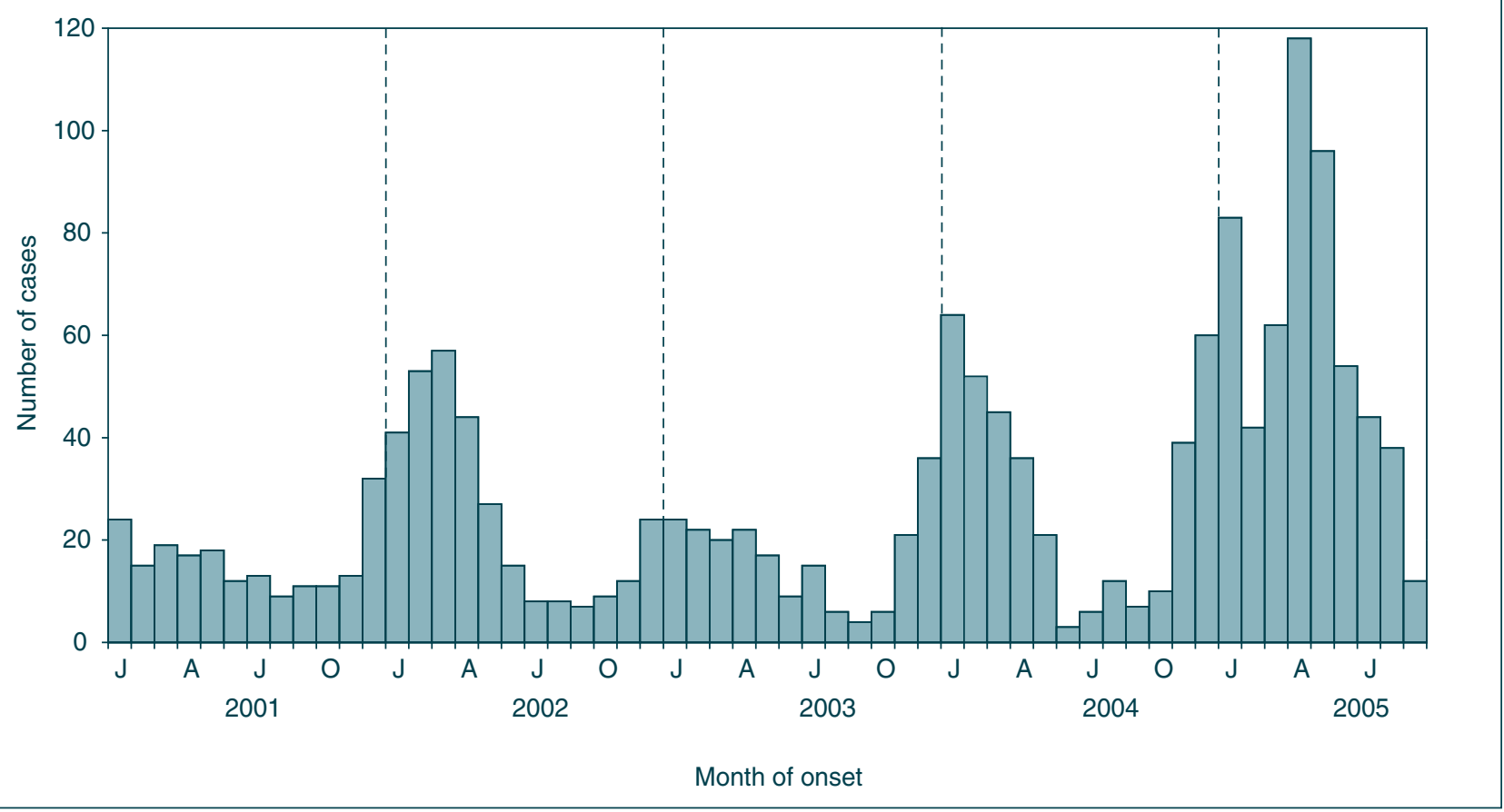

\section{TABLE 1}

CHARACTERISTICS AND REPORTED EXPOSURES OF THE INTERVIEWED CASES OF CRYPTOSPORIDIOSIS NOTIFIED IN NEW SOUTH WALES BETWEEN 1 MAY AND 12 AUGUST 2005, WITH ONSET DATES AFTER 31 MARCH $2005(\mathrm{~N}=178)$

\begin{tabular}{|lc|}
\hline Characteristic or exposure & $\begin{array}{c}\text { Number of cases with characteristic / } \\
\text { Total number of responses* }\end{array}$ \\
\hline Demographic characteristics: & $90 / 178$ \\
Female & $151 / 178$ \\
Resided in Sydney metropolitan area & \\
Reported exposures: & $56 / 165$ \\
Contact with person with diarrhoea & $50 / 175$ \\
Attended childcare centre & $95 / 169$ \\
Swam anywhere & $89 / 169$ \\
Swam in public pool & $142 / 154$ \\
Drank town water supply & $79 / 174$ \\
Contact with animals & \\
\hline
\end{tabular}

\section{TABLE 2}

CRYPTOSPORIDIUM OOCYST LEVELS IN FIVE POOLS THAT HAD MORE THAN TWO REPORTED CASES OF CRYPTOSPORIDIOSIS, SYDNEY, JUNE 2005

\begin{tabular}{|llccc|}
\hline Pool & Locality within Sydney & Date tested & $\begin{array}{c}\text { Number of cases in } 6 \text { weeks } \\
\text { before testing }\end{array}$ & $\begin{array}{c}\text { Cryptosporidium oocyst } \\
\text { count / Litres of water tested }\end{array}$ \\
\hline A & South-West & 3 & $1 / 80$ \\
B & Western & 10 June & $>5$ & $636 / 107$ \\
C & South-West & 17 June & $>3$ & $907 / 100$ \\
D & North & 20 June & $>5$ & Large pool: $1 / 100$ \\
& & 23 June & Small pool: 0/100 \\
\hline
\end{tabular}


Public health unit staff were able to contact 178 of the 254 cases to interview them (or, in the case of children, their families) about possible sources of infection during the exposure period, a follow-up rate of 70 per cent. The median age of the cases was 5.1 years, with a range from 1 month to 57 years. Demographic characteristics and risk factor information are provided for these cases in Table 1.

Eighty-nine cases (53 per cent) reported swimming in a public pool in the 12 days before the onset of symptoms. These cases reported swimming at a total of 35 pools. Of these pools, 22 were named by one case, six were named by two cases, three were named by between three and five cases, and four were named by more than five cases.

\section{Environmental investigation}

Throughout June we identified five swimming pools (at four swimming centres) with more than two associated cases of cryptosporidiosis, and where at least one case had swum in that pool in June within 10 days of onset of illness. Water samples were collected from these pools and tested for the presence of Cryptosporidium oocysts (Table 2).

Public health unit staff contacted these swimming centres to discuss superchlorination practices and review pool disinfectant records. The operators of pools A, B and $\mathrm{D}$ reported that the pools were superchlorinated at least fortnightly. The operator of pool C, which had the highest Cryptosporidium oocyst count, reported the pool had not been superchlorinated for more than six months. There were incomplete superchlorination records at pool $\mathrm{A}$, and there were no records at pools B, C and D.

\section{Laboratory stool testing investigation}

Investigation of changes to Medical Benefits Scheme item numbers relevant to Cryptosporidium stool testing revealed several alterations to the wording for item number 69336 in recent years, with a significant change occurring in May 2003. As a result of this change laboratories could charge against this item using commercial rapid antigen Cryptosporidium detection tests where a faecal concentration was also performed. Previously, private laboratories reported that stools were not necessarily tested for Cryptosporidium unless specifically requested by the doctor because of the laborious nature of detection techniques.

Three NSW diagnostic laboratories were contacted about changes in their stool testing for cryptosporidiosis. The two public laboratories reported no change in the proportion of stools being tested for the presence of Cryptosporidium. The third laboratory, a private laboratory, reported an increased rate of testing for Cryptosporidium as of November 2004, from approximately 10 per cent to 100 per cent of stools. This laboratory notified 56 per cent of the interviewed cases in the period between May and August 2005.

Data obtained from the Medicare Australia website showed that the rates of diagnostic services (per 100,000 population) for the Medical Benefits Scheme item number 69336 in NSW were 760 in 2001-02, 753 in 2002-03, 1004 in 2003-04 and 1013 in 2004-05.

\section{Public health intervention}

In response to the outbreak, NSW Health released a media statement on 10 June 2005 highlighting the increased numbers of cryptosporidiosis cases in the community, and advising swimmers with a diarrhoeal illness to stay out of pools for at least a week after their symptoms resolved. The media release also advised parents on how to prevent Cryptosporidium in swimming pools (for example, by putting waterproof tight fitting pants over young children's swimmers and changing nappies in bathrooms rather than at the poolside).

Public health unit staff contacted swimming centres associated with two or more cases to advise on infection control practices (particularly in toileting areas and near change tables) and disinfectant practices, including superchlorination and shock-dosing. Swimming centres were provided with, and asked to use, 'Keep the cryptobug out of our pool' posters, signage and fact-sheets. Two swimming centres introduced the practice of routinely asking parents if their children had recent or current diarrhoea so that they could be excluded from swimming classes. One public health unit reported contacting local general practitioners to request that they advise the parents of children with diarrhoea that they should not swim in a pool until at least seven days after their symptoms had resolved.

\section{DISCUSSION}

Data from the interviewed cases as well as the pool testing results supports the hypothesis that this outbreak was, at least in part, associated with swimming in contaminated pools.

In NSW, cryptosporidiosis rates tend to rise during the summer months. This outbreak occurred during autumn and winter (April to August 2005), with the majority of cases resident in the Sydney metropolitan area. Of the interviewed cases, 53 per cent reported swimming in a public pool in the 12-day period before the onset of illness. To determine whether this exposure was likely to be associated with illness, we compared our findings with the results of a case-control study by Puech et al $(2001)^{5}$, which investigated a cryptosporidiosis outbreak in NSW in the summer of 1997-98. In the study by Puech et al, 59 per cent of cases reported swimming in a public pool in the exposure period compared with a much lower rate of swimming for controls (38 per cent).

While there are inherent limitations in comparing findings from our investigation with those of Puech et al's study, the results of Puech et al's study provides some evidence that the rate of swimming in a public pool reported by cases at the time of our investigation would have been higher than 
might be expected in a control group (especially as this outbreak occurred during autumn and winter). In Puech et al's study, the reported rates of having attended a childcare centre among controls were similar to the rates of cases in our study (29 per cent in both); however, the cases in our study reported higher rates of having contact with a person with a diarrhoeal illness compared to the controls in Puech et al's study ( 34 per cent vs 25 per cent respectively). This suggests that person-to-person spread may also have played a role in this outbreak.

Laboratory policy and practice can potentially influence notification rates for communicable diseases. There was some evidence to suggest that changes in private laboratory practices may have contributed to the increased number of notifications. While this might explain an overall increase in the rates of cryptosporidiosis in NSW compared with previous years, it does not account for the unusual peak of cases in April to August. Alternatively, increased awareness of cryptosporidiosis within the general population, or increased testing by doctors, may have potentially increased notification rates of cryptosporidiosis. However there was no reason to believe that these factors were contributing to this outbreak.

There are several limitations to this study. Firstly, it is descriptive and therefore cannot provide robust evidence as to causation. However, comparisons of the reported exposures in our cases and those of controls interviewed for a similar outbreak, combined with the findings from the environmental investigation, provides a useful insight into the risk of illness associated with pool swimming in this outbreak.

Secondly, the response rate among cases was 70 per cent. There is no evidence to suggest, however, that the cases we interviewed were significantly different from those who were not. For example, the median age of cases for whom interview information was available was 5.06 years compared with 5.04 years for the entire sample. Eighty-five per cent of interviewed cases were from a metropolitan area compared with 81 per cent for the entire sample. Thirdly, measurement error is likely to have affected the results of pool testing for Cryptosporidium oocysts due to the timelag between reported exposures and pool sampling.

Finally, we were able to contact only three laboratories about changes in their stool testing procedures for cryptosporidiosis. While the private laboratory that accounted for more than 50 per cent of notified cases for this investigation reported a recent increase in their rate of stool testing for cryptosporidiosis, we were not able to obtain data to determine the number of stools tested for cryptosporidiosis from this laboratory in 2005 and previous years, or the number of cryptosporidiosis cases notified from this laboratory over previous years.

Health professionals should routinely provide information to all persons with cryptosporidiosis about the prevention of its spread. The NSW Health fact sheet for cryptosporidiosis specifically advises against swimming while symptomatic and for at least one week afterwards. ${ }^{6}$

To prevent pools being reinfected with Cryptosporidium it is important that pool operators comply with the NSW Health Protocol for minimising the risk of Cryptosporidium contamination in public swimming pools and spa pools. ${ }^{3}$ Pools which have large 'bather loads', especially of young children, should consider increasing the frequency of superchlorination. Other strategies to minimise reinfection in pools includes the use of cryptosporidiosis signage at pools, and asking patrons about recent symptoms of diarrhoea when entering the swimming complex.

\section{ACKNOWLEDGEMENTS}

We would like to acknowledge the assistance of the public health unit staff who undertook interviews with cases, the environmental health officers who investigated pool premises, the NSW Health Water Unit staff who provided timely advice and resources, and Jan Lanser and others who provided information for the laboratory investigation.

\section{REFERENCES}

1. Heymann D (Ed). Control of communicable diseases manual, $18^{\text {th }}$ Edition. Washington: American Public Health Association, 2004.

2. Chen X-M, Keithly J, Paya C, LaRusso N. Current concepts: Cryptosporidiosis. N Engl J Med 2002; 346(22): 1723-31.

3. NSW Health. Protocol for minimising the risk of Cryptosporidium contamination in public swimming pools and spa pools. Sydney: NSW Department of Health, 1999. Available from: www.health.nsw.gov.au/public-health/ehb/ general/pools/cryptopools.pdf

4. Medicare Australia website. www.medicareaustralia.gov. au/providers/health_statistics/statistical_reporting/medicare. htm, accessed 6 January 2006.

5. Puech M, McAnulty J, Lesjak M, Shaw N, Heron L, Watson J. A statewide outbreak of cryptosporidiosis in New South Wales associated with swimming in public pools. Epidemiol Infect 2001; 126: 389-96.

6. NSW Health. Cryptosporidiosis disease. Fact-sheet. 2001. Available from: www.health.nsw.gov.au/infect/diseases. html. 욤 